4. Botros M, Sikaris KA. The de ritis ratio: the test of time. Clin Biochem Rev 2013; 34: 117-30.

5. Hogrel JY, van den Bogaart F, Ledoux I, et al. Diagnostic power of the non-ischaemic forearm exercise test in detecting glycogenosis type $\mathrm{V}$. Eur J Neurol 2015; 22: 933-40.

6. Mastaglia FL, McCollum JP, Larson PF, Hudgson P. Steroid myopathy complicating McArdle's disease. J Neurol Neurosurg Psychiatry 1970; 33: $111-20$.

7. Baker SK, Vladutiu GD, Peltier WL, Isackson PJ, Tarnopolsky MA. Metabolic myopathies discovered during investigations of statin myopathy. Can J Neurol Sci 2008; 35: 94-7.

8. García-Consuegra I, Rubio JC, Nogales-Gadea G, et al. Novel mutations in patients with McArdle disease by analysis of skeletal muscle mRNA. J Med Genet 2009; 46: 198-202.

9. Bruno C, Cassandrini D, Martinuzzi A, et al. McArdle disease: the mutation spectrum of PYGM in a large Italian cohort. Hum Mutat 2006; 27: 718

10. Wu L, Brady L, Shoffner J, Tarnopolsky MA. Next-generation sequencing to diagnose muscular dystrophy, rhabdomyolysis, and hyperCKemia. Can J Neurol Sci 2018; 45: 262-8.

11. Landsverk ML, Douglas GV, Tang S, et al. Diagnostic approaches to apparent homozygosity. Genet Med 2012; 14: 877-82.

DOI: https://doi.org/10.1016/j.pathol.2020.09.019

\section{EBV positive fibrin/chronic inflammation associated diffuse large B-cell lymphoma: an incidental finding associated with a breast implant}

Sir,

We report a rare case of Epstein-Barr virus positive $(\mathrm{EBV}+)$ fibrin/chronic inflammation associated large B cell lymphoma (FA-DLBCL) occurring in association with a textured breast implant.

A 70-year-old well woman presented for removal of a right-sided breast implant after a 4 month history of minor pain and capsular contracture. The Allergan macrotextured silicone implant had been in situ for 9 years, initially placed as part of a breast reconstruction following mastectomy treatment alone for a primary breast carcinoma. En bloc surgical removal of the intact implant and capsulectomy was performed without any prior biopsy or fine needle aspiration procedure. During specimen grossing, yellow-cream fluid was present between the internal layer of the fibrous capsule and the surface of the implant (Fig. 1A). The capsule was slightly thickened without discrete masses. Systemic work-up showed no other sites of disease including a normal lactate dehydrogenase (LDH) level. There was no history of prior lymphoma or systemic immunosuppression. A diagnosis of EBV + FA-DLBCL was rendered after incorporating the clinical and radiological findings. Surgical removal of the implant alone was performed. There is no evidence of disease progression, recurrence or systemic lymphoma after one year of close clinical follow-up and interval PET imaging.

Microscopic examination of the capsule showed clusters and aggregates of atypical large pleomorphic cells, localised to the space between the breast capsule and implant. The cells were mostly enmeshed within fibrin on the inner aspect of the capsule and also very focally within the fibrous capsule (Fig. 1B,C). There was no angiocentric growth, vascular wall invasion or destruction.
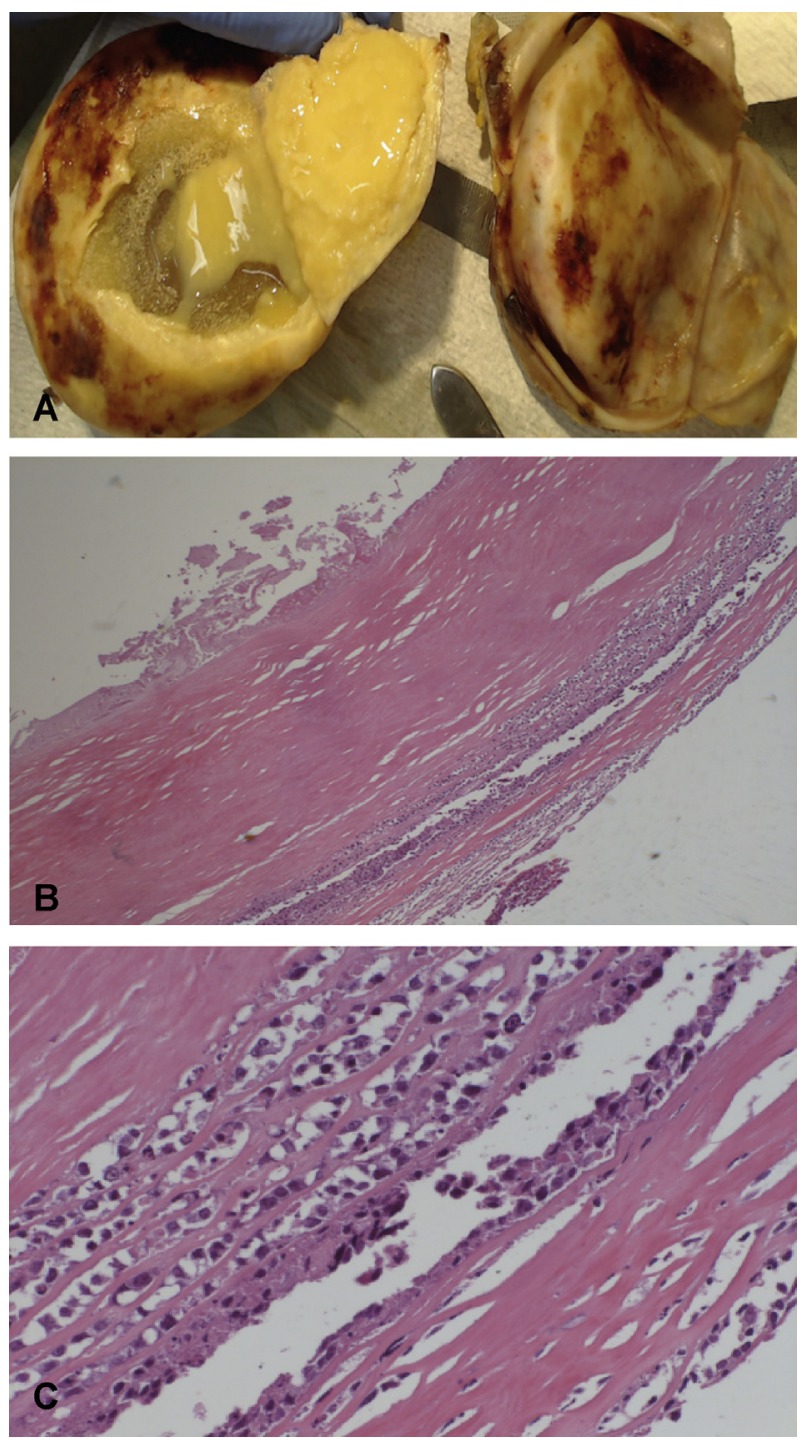

Fig. 1 (A) Thickened yellow fluid present in the space between the fibrous capsule and surface of the implant. (B,C) Aggregates of pleomorphic large cells within the fibrin and fibrous capsule.

The atypical cells expressed CD45, CD79a (Fig. 2A), CD20, PAX5, CD30, BCL6, MUM1, PD-L1 (SP142 clone) and were negative for CD10, pan-T-cell markers (CD3, CD2, CD4, CD5, CD7, CD8), cytotoxic markers (TIA1, Perforin, Granzyme B), ALK, CD68, carcinoma markers (AE 1/AE3, Cam 5.2, EMA) and for HHV-8 latent nuclear antigen-1 (Fig. 2B,D). Only small numbers of CD3/CD8 coexpressing reactive small $\mathrm{T}$ cells were present in the background. Ki-67 was confined to the pleomorphic large cells. The majority of cells harboured EBV, demonstrated by in situ hybridisation (Fig. 2E). LMP1 and EBNA2 were not available in our laboratory for latency typing. Immunoglobulin heavy chain rearrangement by polymerase chain reaction (PCR) detected a monoclonal population. There was no evidence of $M Y C$ gene rearrangement, copy number variation or amplification. Next generation sequencing was attempted but did not yield a result due to the nature of the DNA.

FA-DLBCL is a provisional clinicopathological entity in the 2017 World Health Organization (WHO) Classification, 

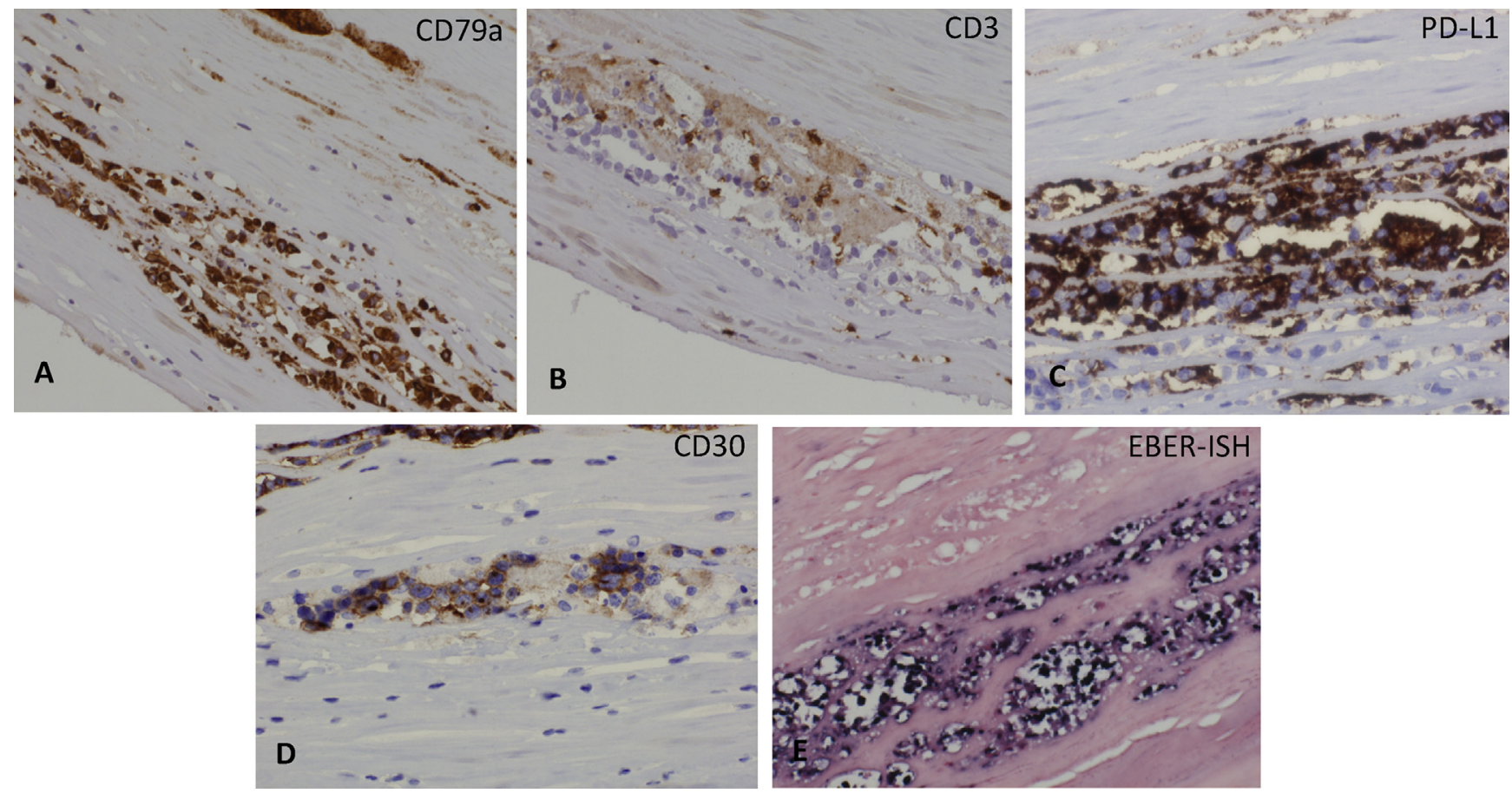

Fig. 2 (A) The tumour cells showed strong expression for B cell markers CD79a, CD20, Pax5. (B) The tumour cells were negative for pan-T-cell markers. (C) The majority of the tumour cells showed strong PD-L1 expression (SP142 clone). (D) The tumour cells are strongly positive for CD30 and (E) EBER-ISH.

listed under the category of diffuse large B-cell lymphoma associated with chronic inflammation (DLBCL-CI). DLBCL$\mathrm{CI}$, occurs in the context of long-standing chronic inflammation, shows association with EBV, with pyothorax associated lymphoma (PAL) being the archetype of this category. ${ }^{1}$ In PAL, patients are symptomatic, with elevated LDH levels. It pursues an aggressive clinical course and at the molecular level has been reported to harbour a high degree of genetic complexity with complex karyotypes (numerical chromosomal abnormalities) including p53 mutation, $M Y C$ amplification and TNFAIP3 deletion. ${ }^{2}$

Rare cases of DLBCL-CI mimicking PAL have also been reported at other anatomical sites preceded by long-standing chronic inflammation such as in association with metallic implants for orthopaedic procedures, surgical mesh implant and long standing chronic venous cutaneous ulcer. ${ }^{3}$ These mass-forming lesions associated with a foreign body or longstanding chronic inflammation appear to conform to or share overlapping features with DLBCL-CI, despite a more indolent clinical course, suggesting perhaps this is a distinctive clinical entity ${ }^{4,5}$ more akin to other EBV-associated B cell lymphoproliferation than 'lymphoma'.

In addition, in recent years there has been an increasing number of case reports and case series describing incidentally discovered microscopic $\mathrm{EBV}+\mathrm{B}$ cell proliferations arising in a variety of unusual sites, such as in pre-existing cystic lesions and pseudocysts (splenic, renal pseudocysts, adrenal, testicular hydrocele), in the cardiovascular system (atrial myxomas, atrial thrombus, replacement prosthetic cardiac valves) and in association with chronic subdural haematomas, haematomas or thrombi associated with sites of prior vascular surgery. ${ }^{5-7}$ The reported cases occur in the absence of overt tumour nodules or masses, show no invasion into adjacent tissue structures, lack suppurative inflammation, are of localised stage, and occur as incidental findings in immunocompetent individuals. Common to all is the microscopic B-cell lymphoproliferation within aggregated fibrin/ fibrinous material, enclosed within a restricted space with low tumour burden. The atypical lesional cells have been described as growing along the wall of the pre-existing sclerotic wall of a cystic space or cavity. ${ }^{5}$ The reported cases share similar immunomorphology to our case in addition to demonstrable clonal immunoglobulin gene rearrangement on polymerase chain reaction (PCR) and no $M Y C$ rearrangement.

Treatments vary from surgical removal alone to those with the addition of radiation therapy, rituximab or standard cycles of RCHOP chemotherapy. Regardless of the therapeutic modality, in the largest series to date by Boyer et al., ${ }^{5}$ all cases (strictly defined as incidental findings of lesional cells within conspicuous fibrin without an associated mass lesion) have been associated with excellent outcomes. Recurrent or persistent disease was seen in a small subset of cases in which there was primary cardiac or vascular involvement, suggesting persistence of disease when occurring in sites that are less likely to be amenable to complete surgical removal.

DLBCL-CIs are postulated to arise at the site of chronic inflammation, driven by local immunosuppression. The cytokines produced are thought to play an important role in lymphomagenesis by enabling EBV-infected B cells to evade immune surveillance and stimulate cell growth. ${ }^{8}$ The large pleomorphic EBV infected cells show a type III latency pattern, with expression of EBNA2, LMP1 and BZLF1, ${ }^{5}$ with this latency program occurring only during acute EBV infection or in immunodeficiency states. ${ }^{4}$ The tumour cells have also been reported to show high PD-L1 expression. ${ }^{5}$ PD-L1 expression has been reported in tumour cells of some aggressive B-cell lymphomas and virus- and immunodeficiency-associated malignancies, associated with an ineffective $\mathrm{T}$ cell immune response to tumours. ${ }^{9}$ It is 
thought that the compounding effect of local immunosuppression and physical barrier in an enclosed environment allows the EBV infected $\mathrm{B}$ cells to evade immunosurveillance and proliferate in an 'immune privileged' environment (due to absence of EBV specific cytotoxic T cells." The HIF-1A expression supports the hypoxic state induced by the walled off space.

The morphology and immunophenotype of the lesional cells in FA-DLBCL or CI-DLBCL can overlap with other EBV associated B cell lymphomas or lymphoproliferative disorders listed in the WHO classification, such as plasmablastic lymphoma, EBV+ DLBCL NOS, EBV-associated mucocutaneous ulcers, lymphomatoid granulomatosis and post-transplant lymphoproliferative disorders (PTLD). ${ }^{1}$ All these entities have in some ways arisen from reduced immune surveillance for EBV, either from iatrogenic immunosuppression (e.g., medications, post-transplant setting), aging and immune senescence, or primary or hereditary immune deficiency.

The occurrence of this current lymphoma adjacent to a breast implant, raises the important pitfall for potential misclassification as breast implant-associated anaplastic large cell lymphoma (BI-ALCL). BI-ALCL is a new provisional entity in the revised 2017 WHO classification of lymphoid malignancies of $\mathrm{T}$ cell lineage, arising in association with a breast implant, usually as an effusion confined to the surrounding fibrous capsule or less often as a mass or axillary lymphadenopathy. ${ }^{1}$ The pathogenesis of BI-ALCL is not well delineated with several plausible associations including chronic inflammation, textured implants and bacterial biofilm infection. ${ }^{10} \mathrm{~A}$ recent gene expression profiling study demonstrated a hypoxia signature suggesting this lymphoma arises in a unique microenvironment. ${ }^{11}$ Mutations affecting the JAK-STAT signalling pathway and alterations in epigenetic modifiers have been identified in BI-ALCL. ${ }^{12}$ Of note, our current case occurred in association with a macrotextured implant, which is more likely associated with the development of BI-ALCL. In our case, the initial $H \& E$ impression may have led to a misdiagnosis of BI-ALCL if only a limited panel of immunohistochemistry was performed or this diagnosis was not considered. BI-ALCLs are of $\mathrm{T}$ cell lineage as opposed to $\mathrm{B}$ cell origin in FA-DLBCL. Both tumours (BI-ALCL and FA-DLBCL) are MUM1 and CD30 positive. The anaplastic large cells of BIALCL usually show loss of pan-T-cell markers, and tumour cells of the FA-DLBCL showing a post-germinal centre phenotype may lose CD20 expression.

Here we report a unique case of EBV + FA-DLBCL arising as an incidental finding in a breast implant capsulectomy specimen, expanding the geographical sites in which such cases have been reported. The similarities in its clinical presentation to BI-ALCL highlights the potential misclassification of this entity if it is not considered diagnostically. The cells of FA-DLBCL morphologically mimic other aggressive EBV-driven B cell lymphomas but the clinical presentation and host factors aid in the distinction, highlighting the importance of integrating the clinical and radiological/staging features in arriving at this diagnosis. As highlighted in the literature to date, this entity is more akin to a lymphoproliferation than a lymphoma.

Conflicts of interest and sources of funding: The authors state that there are no conflicts of interest to disclose.
Christine Khoo ${ }^{1}$, Christine McTigue ${ }^{2}$, David J. HunterSmith $^{3}$, Patricia Walker ${ }^{4}$

${ }^{1}$ Department of Pathology, Peter MacCallum Cancer Centre, Melbourne, Vic, Australia; ${ }^{2}$ Dorevitch Pathology, Melbourne, Vic, Australia; ${ }^{3}$ Department of Plastic and Reconstructive Surgery, Peninsula Health, Melbourne, Vic, Australia; ${ }^{4}$ Department of Haematology, Peninsula Health, Peninsula Private Hospital, and Alfred Health, Melbourne, Vic, Australia

Contact Dr Christine Khoo.

E-mail: Christine.khoo@petermac.org

1. Swerdlow SH, Campo E, Harris NL, et al., editors. WHO Classification of Tumours of Haematopoietic and Lymphoid Tissues. Revised 4th ed. Lyon: IARC, 2017.

2. Aozasa K, Takakuwa T, Nakatsuka S. Pyothorax-associated lymphoma, a lymphoma developing in chronic inflammation. Adv Anat Pathol 2005; 12: 324-31.

3. Lymphoma P, Cheuk W, Chan ACL, et al. Metallic implant-associated lymphoma. Am J Surg Pathol 2005; 29: 832-6.

4. Dojcinov S, Fend F, Quintanilla-Martinez L. EBV-positive lymphoproliferations of B- T- and NK-cell derivation in nonimmunocompromised hosts. Pathogens 2018; 7: 28.

5. Boyer DF, McKelvie PA, De Leval L, et al. Fibrin-associated EBV positive large b-cell lymphoma. Am J Surg Pathol 2017; 41: 299-312.

6. Gruver AM, Huba MA, Dogan A, Hsi ED. Fibrin-associated large Bcell lymphoma: Part of the spectrum of cardiac lymphomas. Am J Surg Pathol 2012; 36: 1527-37.

7. Boroumand N, Ly TL, Sonstein J, Medeiros LJ. Microscopic diffuse large B-cell lymphoma (DLBCL) occurring in pseudocysts. Am J Surg Pathol 2012; 36: 1074-80.

8. Kanno H, Naka N, Yasunaga Y, et al. Production of the immunosuppressive cytokine interleukin-10 by Epstein-Barr-virus-expressing pyothorax-associated lymphoma: possible role in the development of overt lymphoma in immunocompetent hosts. Am J Pathol 1997; 150: 349-57.

9. Chen BJ, Chapuy B, Ouyang J, et al. PD-L1 expression is characteristic of a subset of aggressive B-cell lymphomas and virus-associated malignancies. Clin Cancer Res 2013; 19: 3462-73.

10. Quesada AE, Medeiros JL, Clemens MW, Ferrufino-Schmidt MC, PinaOviedo S, Miranda RN. Breast implant-associated anaplastic large cell lymphoma: a review. Mod Pathol 2019; 32: 166-88.

11. Oishi N, Hundal T, Phillips JL, et al. Molecular profiling reveals a hypoxia signature in breast implant-associated anaplastic large cell lymphoma. Haematologica 2020; May 15: haematol.2019.245860.

12. Laurent C, Nicolae A, Laurent C, et al. Gene alterations in epigenetic modifiers and JAK-STAT signaling are frequent in breast implantassociated ALCL. Blood 2020; 135: 360-70.

DOI: https://doi.org/10.1016/j.pathol.2020.09.022

\section{Pembrolizumab associated sarcoid-like lymphadenopathy mimicking progressive disease in Hodgkin lymphoma}

Sir,

In recent years, the use of programed cell death-1 (PD-1) checkpoint inhibitors such as pembrolizumab and nivolumab has emerged as a treatment modality in patients with various malignancies such as melanoma, non-small cell lung cancer, squamous cell carcinoma of the head and neck, urothelial carcinoma, microsatellite instability or mismatch repair deficient metastatic colorectal cancer and relapsed/refractory Hodgkin lymphoma (HL). ${ }^{1}$ In HL, these treatments work via 